menstruation ; in the other, who has a congested and tender left ovary, the left parotid gland does not secrete during menstruation, and then the mouth and fauces on that side are dry and painful."

CASE 60.-Billroth, among his Zurich cases, had one of parotitis after a blow on the testicle.

\section{STONE SACCULATED IN THE BLADDER OF A FEMALE.}

By CHAS. WILLIAMS, F.R.C.S. ED., SURGHON TO THE NORFOLK AND NORWICR HOSPITAL.

CASES in which a vesical calculus is impacted in a cyst situated in the walls of the bladder are so extremely rare, that I consider it a duty to record this very interesting example.

A fine and healthy girl, aged three years, living in Norwich, came under the care of the late Mr. George Hutchison in the year 1873, having for several months previously suffered from very decided symptoms of stone in the bladder. It had been noticed by her mother that from the time of her birth she had experienced a difficulty, as well as occasionally severe pain in passing urine, and that sometimes she voided blood mixed with it, and was in the habit of straining so violently as to produce prolapsus of the rectum.

On sounding the bladder, which was an unusually capacious one, it was with some difficulty that a calculus could be detected. At the wish of the parents Mr. Hutchison resolved to remove the stone by dilatation. Mr. W. H. Day assisted at the operation, and I was requested to administer chloroform. The urethra was freely and quickly dilated with Weiss's trivalve dilator. There was considerable trouble to find the stone, and when found a still greater trouble to seize it with she forceps (and it was particularly noticed that, although the patient was thoroughly under the influence of the anæsthetic, the getting hold of the stone with the forceps occasioned severe straining); the blades could not be made to grip the calculus; they continually slipped off, bringing away pieces of the stone. At last it became absolutely necessary to ascertain what occasioned the difficulty. For this purpose the urethra was still further dilated, and the neck of the bladder was also divided with a probe-pointed bistoury. The stone could now be felt with the point of the finger immovably fixed in the floor of the bladder on the patient's left. It appeared to be of the size of a pigeon's egg, and was enclosed in a sac, through the neck of which a small portion protruded into the vesical cavity, and it was off this nodule that the forceps so continuously slipped. Many efforts were made to dislodge itfirst with a scoop, then with the finger, which could barely reach it, and next with the forceps; they all proved unsuccessful. Several portions were broken off the uncovered portion, but the main prece was left in situ, as it was considered undesirable to make any further attempt to remove it, the patient having been a long time under the influence of chloroform and apparently in a very exhausted condition.

The next day the child had voided very little urine. A catheter was introduced, and a small quantity of sanguineous urine flowed out. She was very drowsy, and had been so since the operation. When roused she took milk and brandy very freely, but immediately afterwards became drowsy again; she did not appear to have recovered from the influence of the chloroform. The next day she died. No post-mortem examination was permitted.

I am induced to believe this child died of chronic chloroform-poisoning, and not from the effects of the operation, which was by no means roughiy performed, and there was very little blood lost. She never thoroughly revived, but became comatose, and died in that condition. It is difficult to imagine what could have given rise to the formation of the sac; there never was an obstruction to the escape of the urine, such as stricture or prostatic enlargement might engender, for neither existed. We are taught that a cyst is usually formed by the straining necessary to expel the urine; the mucous membrane is forced between the bands of muscular fibres, hypertrophied in consequence of the strain to which they are subjected. Nothing of the sort can apply in this case, and it is not easy to believe that the stone was the cause of the cyst, which it might have been had it been situated close to the neck of the bladder. When impacted in this situation, the very pressure to which a stone is subjected by the constant and long-continued action of the bladder to expel it causes the mucous membrane to ulcerate tlirough, and the stone is in due time forced into a carity, which enlarges as the stone grows, and in this way it may form a tumour in the vagina, as was the case reported by me in THE LANCET of Nov. 7th, 1885. An effort is then made by nature to contract the opening, which in this child was nearly accomplished; but the calculus was far from the neck of the bladder, and could barely be touched with the point of the finger, so that a different explanation of the formation of the cyst is required; and as no examination was allowed to be made, it seems to me to be almost impossible to suggest in what way the sac was formed. Sabulous matter or a few urinary crystals may probably have been deposited originally in a mucous follicle, lacuna, or fossa, and gradually augmented in quantity, and in this way the sac enclosing the calculus may have been produced. The mother of the girl at four years of age suffered from stone, which was removed by the late Dr. Edward Lubbock; it was the size and shape of a walnut. She has suffered from incontinence since that time.

\section{IMPERFORATE RECTUM TREATED SUCCESS-} FULLY BY LEFT INGUINAL COLOTOMY.

$$
\text { Br HUGH STOTT, M.R.C.S., L.S.A.L. }
$$

A CASE of imperforate rectum was recently brought under my care. The subject was a small but full-time infant four days old; it was an illegitimate child. The mother had noticed that the baby had not passed a motion since birth, and that it was constantly sick, the vomit being of a yellow colour, of a strong odour, and staining the clothes. The mother at the birth of the child was attended by a midwife. The rectum, on examination, disclosed a natural opening for the anus, and on passing a bougie made of soap up the canal it was arrested at the second inch; by carefully inserting the little finger no bulging of bowel or any indication of such could be made out. I therefore resolved, instead of dissecting up and seeking the end of the bowel, to perform left inguinal colotomy or Littrés operation. For this purpose I gave chloroform, and made an incision above Poupart's ligament in the left groin, about two inches in length, in a curved direction upwards towards the anterior superior spine of the ilium. Upon opening the peritoneum a quantity of yellow-coloured fluid exuded, and the large bowelt bulged forwards; this was pushed upwards until the lowest part was reached. It had no free end, but was attached. The bowel was stitched on either side of the edges of the wound and then opened between the stitches. Some meconium escaped; there was little or no bleeding. The wound was dressed with strips of lint saturated with carbolised oil ( 1 in 100), a loose pad of cotton-wool, and then a bandage. The child recovered from the chloroform and was not sick; it was put to bed with its mother, and took from the bottle cow's milk and water. The stitches, which were silk; were removed on the third day, and the wound healed kindly, without any sign of suppuration which is to be wondered at considering the wretched and dirty state of the surroundings. The child is now three months old, and is thriving; the wound has perfectly healed, and looks clean and healthy; at present there is no prolapse of bowel. The operation was done by myself without assistance, except that an old woman looked on. This will show the simplicity of the operation.

Perhaps the question may be raised, Was it right to endeavour to prolong this child's life? It has been said in such cases, "Let the child die," but I strongly agree with Mr. T. Holmes, who says, in reference to these cases, "A surgeon who would not operate in a case of this kind would I think, neglect one of the plainest duties of his profession." Is a surgeon to arrogate the power as to whether a person shall live or die? As to the choice of operations, I selected Littrês : first, because it is easier to do without assistance: and, secondly, because contractions following a dissection upwards in the course of the rectum are so liable to take place, leaving the patient probably in a worse state than he was before. It may be $I$ shall later on endearour to establish a natural discharge for the freal matter at the anus. 